\title{
EXACT DIAGONALIZATION OF THE $d$-DIMENSIONAL SPATIALLY CONFINED QUANTUM HARMONIC OSCILLATOR
}

\author{
Kunle Adegoke*, Adenike Olatinwo, Henry Otobrise, Funmi Akintujoye, \\ Afees Tiamiyu \\ Department of Physics and Engineering Physics, Obafemi Awolowo University, \\ 220005 Ile-Ife, Nigeria \\ *Corresponding author, E -mail: adegoke00@gmail.com
}

(Received April 8, 2016)

\begin{abstract}
In the existing literature various numerical techniques have been developed to quantize the confined harmonic oscillator in higher dimensions. In obtaining the energy eigenvalues, such methods often involve indirect approaches such as searching for the roots of hypergeometric functions or numerically solving a differential equation. In this paper, however, we derive an explicit matrix representation for the Hamiltonian of a confined quantum harmonic oscillator in higher dimensions, thus facilitating direct diagonalization.
\end{abstract}

Keywords: matrix, Hamiltonian, quantum harmonic oscillator, direct diagonalization.

\section{INTRODUCTION}

The $d$-dimensional confined harmonic oscillator (cho) of mass $m$ and frequency $\omega$ is described by the Hamiltonian

$$
H(\mathbf{x})=-\frac{\hbar^{2}}{2 m} \nabla^{2}+\frac{1}{2} m \omega^{2} \mathbf{x}^{2},
$$

where $\mathbf{x}=\left(x_{1}, x_{2}, \ldots, x_{d}\right)$ with $\left|x_{i}\right| \leq L, \mathbf{x}^{2}=\mathbf{x}^{t} \mathbf{x}$ and $\nabla$ is the $d$-dimensional cartesian gradient operator. $H(\mathbf{x})$ being a Kronecker sum, we can also write

where

$$
H(\mathbf{x})=\sum_{i=1}^{d} H_{i}\left(x_{i}\right),
$$

$$
H_{i}\left(x_{i}\right)=-\frac{\hbar^{2}}{2 m} \frac{\partial^{2}}{\partial x_{i}^{2}}+\frac{1}{2} m \omega^{2} x_{i}^{2}, \quad\left|x_{i}\right| \leq L .
$$

We also note that $H(\mathbf{x})=T(\mathbf{x})+V(\mathbf{x})$, where

with

$$
T(\mathbf{x})=\sum_{i=1}^{d} T_{i}\left(x_{i}\right) \text { and } V(\mathbf{x})=\sum_{i=1}^{d} V_{i}\left(x_{i}\right),
$$

$$
T_{i}\left(x_{i}\right)=-\frac{\hbar^{2}}{2 m} \frac{\partial^{2}}{\partial x_{i}^{2}} \text { and } V_{i}\left(x_{i}\right)=\frac{1}{2} m \omega^{2} x_{i}^{2}, \quad(i=1,2, \ldots, d)
$$


Various techniques have been employed by researchers to numerically diagonalize the Hamiltonian of a confined oscillator. These methods usually involve searching for roots of hypergeometric functions, as can be seen for example in references (AL-JABER, 2008) and (MONTGOMERY et al. 2010). In (CAMPOY et al. 2002) a method based on the expansion of the wavefunction as well as numerical integration of an ordinary differential equation were used to obtain the energy eigenvalues and wavefunctions of a one-dimensional confined oscillator.

In this paper we will derive an explicit matrix representation for the Hamiltonian of the confined $d$-dimensional harmonic oscillator.

\section{MATRIX REPRESENTATION OF THE OPERATORS}

If we consider each operator $T_{i}\left(x_{i}\right)$ as living in an $N$ - dimensional Hilbert space, then the functions

$$
\varphi_{r}\left(x_{i}\right)=\sqrt{\frac{1}{L}} \cos \left[\frac{\pi}{2} \sin ^{2}\left(\frac{r \pi}{2}\right)-\frac{(r+1) \pi x_{i}}{2 L}\right], \quad r=0,1,2, \ldots, N-1,
$$

constitute a set of basis vectors of this $N$ - dimensional Hilbert space since they are the non-degenerate, mutually orthogonal and normalized eigenstates of the Hermitian operator $T_{i}$, with corresponding eigenvalues

$$
\varepsilon_{r}=(r+1)^{2} \varepsilon, \quad r=0,1,2, \ldots, N-1, \text { where } \varepsilon=\frac{\pi^{2} \hbar^{2}}{8 m L^{2}} .
$$

Thus the operator $T(\mathbf{x})$ lives in an $N^{d}$ dimensional Hilbert space whose basis vectors can be taken as the direct product vectors

$$
\psi_{s}(\mathbf{x})=\psi_{s}\left(x_{1}, x_{2}, \ldots, x_{d}\right)=\prod_{i=1}^{d} \varphi_{s_{i}}\left(x_{i}\right), \quad s=0,1,2, \ldots, N^{d}-1,
$$

where

$$
s_{i}=\left\lfloor\frac{s}{N^{d-i}}\right\rfloor \bmod N, \quad i=1,2, \ldots, d,
$$

where $\lfloor q\rfloor$, the floor of $q$, is the nearest integer not greater than $q$.

Thus each state $\psi_{s}(\mathbf{x})$ is uniquely characterized or labelled by a vector $\mathbf{s}=\left(s_{1}, s_{2}, \ldots, s_{d}\right)$ such that $s_{i} \in[0,1,2, \ldots, N-1]$.

Denoting the eigenvalues of $T(\mathbf{x})$ by $e_{s}$, we have

$$
e_{s}=\sum_{i=1}^{d} \varepsilon_{s_{i}}=\varepsilon \sum_{i=1}^{d}\left(s_{i}+1\right)^{2}, \quad s=0,1,2, \ldots, N^{d}-1,
$$

with $s_{i}$ as defined in (4) and $\varepsilon$ as given in (2).

Since the cho Hamiltonian $H(\mathbf{x})$ lives in the same Hilbert space as $T(\mathbf{x})$, the complete set of functions $\left\{\psi_{s}\right\}$, with $\psi_{s}$ as given in (3), will be used as the basis vectors for the matrix representation of $H$.

Thus, for $s=0,1,2, \ldots, N^{d}-1$ and $t=0,1,2, \ldots, N^{d}-1$ and with $s_{i}$ and $t_{i}$ as given in (4), the $N^{2 d}$ matrix elements of $H$ are given by 


$$
\begin{aligned}
H_{s t} & =\left\langle\psi_{s}|H| \psi_{t}\right\rangle \\
& =\sum_{i=1}^{d}\left\langle\psi_{s}\left|H_{i}\right| \psi_{t}\right\rangle \\
& =\sum_{i=1}^{d}\left\{\prod_{j=0}^{d-1}\left\langle\varphi_{s_{d-j}}\left(x_{d-j}\right)\left|H_{i} \prod_{j=1}^{d}\right| \varphi_{t_{j}}\left(x_{j}\right)\right\rangle\right\} \\
& =\sum_{i=1}^{d}\left\{\left(\prod_{j=1}^{d} \delta_{j \neq i}\right)\left(\left\langle\varphi_{s_{i}}\left|H_{i}\right| \varphi_{t_{i}}\right\rangle\right)\right\} \\
& =\sum_{i=1}^{d} c_{i_{s t}} H_{i_{s_{i} t_{i}}},
\end{aligned}
$$

where we have introduced a $d$-dimensional vector c whose components are $N^{d} \times N^{d}$ symmetric binary matrices, $c_{i}$ with elements given by

$$
c_{i_{s t}}=\prod_{j=1}^{d} \delta_{s_{i} t_{i}},
$$

so that $c_{i_{s t}}=1$ if either the two vectors $\mathbf{s}$ and $\mathbf{t}$ are one and the same vector, $\mathbf{s}=\mathbf{t}$, or they differ only at the $i^{\text {th }}$ component, otherwise $c_{i_{s t}}=0$.

We note that

$$
\delta_{s_{i} t_{i}} c_{i_{s t}}=\delta_{s t} \text {. }
$$

In (5), $H_{i}, i=1,2, \ldots d$ are $N^{d} \times N^{d}$, symmetric matrices with elements

$$
\begin{aligned}
H_{i_{s_{i} t_{i}}} & =\left\langle\varphi_{s_{i}}\left|H_{i}\right| \varphi_{t_{i}}\right\rangle \\
& =\left\langle\varphi_{s_{i}}\left|T_{i}\right| \varphi_{t_{i}}\right\rangle+\left\langle\varphi_{s_{i}}\left|V_{i}\right| \varphi_{t_{i}}\right\rangle \\
& =T_{s_{i} t_{i}}+V_{i_{i_{i} t_{i}}},
\end{aligned}
$$

so that (5) can now be written as $H_{s t}=T_{s t}+V_{s t}$ with

$$
T_{s t}=\sum_{i=1}^{d} c_{i_{s t}} T_{i}, \quad V_{s t}=\sum_{i=1}^{d} c_{i_{s t}} V_{i_{s_{i} t_{i}}}
$$

We introduce yet another $d$-dimensional vector, $\boldsymbol{\alpha}$, whose components, $\alpha_{i}$, are $N^{d} \times N^{d}$ symmetric binary matrices, in terms of which the $c_{i}$ matrices may also be expressed. The $\alpha_{i}$ matrices are defined through their elements by $\alpha_{i_{s t}}=\delta_{s_{i} t_{i}}$.

\section{Properties of the auxilliary matrices $c_{i}$ and $\alpha_{i}$}

It is straightforward to verify the following property for the $\alpha_{i}$ matrices:

$$
\alpha_{i} \alpha_{j}=\alpha_{j} \alpha_{i}=N^{d-1} \delta_{i j} \alpha_{i}+N^{d-2}\left(1-\delta_{i j}\right) J_{N^{d}},
$$

where

$$
J_{N^{d}}=\left(\begin{array}{cccc}
1 & 1 & \vdots & 1 \\
1 & 1 & \vdots & 1 \\
\vdots & \vdots & \vdots & \vdots \\
1 & 1 & \vdots & 1
\end{array}\right)
$$


is the $N^{d} \times N^{d}$ all-ones matrix. The $\alpha_{i}$ matrices are singular and have trace equal to $N^{d}$. The eigenvalues of $\alpha_{i}$ are $N^{d-1}$ repeated $N$ times and 0 repeated $N^{d}-N$ times. Finally using multinomial expansion theorem and (10), it is readily established that the matrix $\alpha=\sum_{i=1}^{d} \alpha_{i}$ satisfies

$$
\alpha^{2}=N^{d-1} \alpha+N^{d-2} d(d-1) J_{N^{d}}
$$

From (6) it follows that

$$
c_{i_{s t}}=\delta_{s t}+\left(1-\alpha_{i_{s t}}\right) \delta_{\alpha_{s t}, d-1}
$$

and

Explicitly

$$
c_{s t}=\sum_{i=1}^{d} c_{s t}=\delta_{s t} d+\delta_{\alpha_{s t}, d-1}
$$

$c_{i_{s t}}=\left\{\begin{array}{cc}\cos ^{2}\left(\alpha_{i_{s t}} \pi / 2\right) & \text { if } \alpha_{s t}=d-1 \\ 0 & \text { if } \alpha_{s t}<d-1 \\ 1 & \text { if } s=t\end{array}\right.$

and

$c_{s t}= \begin{cases}0 & \text { if } \alpha_{s t}<d-1 \\ 1 & \text { if } \alpha_{s t}=d-1 \\ d & \text { if } s=t\end{cases}$

From the definition of the $c_{i}$ matrices the following further properties are evident:

1. $c_{i}^{n}=N^{n-1} c_{i}$, for $n \in \mathrm{Z}^{+}$.

2. The eigenvalues of $c_{i}$ are 0 and $N$, each being $N^{d-1}$ - fold degenerate.

3. The $c_{i}$ matrices are singular and have trace $N^{d}$.

\section{Representation for $T$}

Since $T_{i}=\left\langle\varphi_{s_{i} t_{i}}\left|T_{i}\right| \varphi_{t_{i}}\right\rangle$, from (9) we have

$$
\begin{aligned}
T_{s t} & =\sum_{i=1}^{d} c_{i_{s t}} \varepsilon\left(s_{i}+1\right)^{2} \delta_{s_{i} t_{i}} \\
& =\varepsilon \delta_{s t} \sum_{i=1}^{d}\left(s_{i}+1\right)^{2},
\end{aligned}
$$

where we have used (1), (2) and (7). 


\section{Representation for $V$}

Since $V_{s_{i} t_{i}}=\left\langle\varphi_{s_{i}}\left|V_{i}\right| \varphi_{t_{i}}\right\rangle$, performing the indicated integrations, with $\varphi_{s_{i}}$ and $\varphi_{t_{i}}$ as given in (1) and noting that the only non-zero matrix elements of $V_{i}$ are those for which $s_{i}$ and $t_{i}$ are of the same parity, we obtain

$$
\begin{aligned}
V_{i_{s_{i} t_{i}}}=\frac{\lambda^{2} \varepsilon}{2} & {\left[\frac{\left(1-\delta_{s_{i} t_{i}}\right)}{\left(s_{i}-t_{i}\right)^{2}+\delta_{s_{i} t_{i}}}-\frac{\left(1-\delta_{s_{i} t_{i}}\right)}{\left(s_{i}+t_{i}+2\right)^{2}}\right] \cos ^{2}\left[\left(s_{i}-t_{i}\right) \frac{\pi}{2}\right] } \\
+ & \frac{\lambda^{2} \varepsilon}{8} \delta_{s_{i} t_{i}}\left[\frac{\pi^{2}}{6}-\frac{1}{\left(s_{i}+1\right)^{2}}\right]
\end{aligned}
$$

where $\lambda=\omega \hbar / \mathcal{E}=\varepsilon_{\omega} / \mathcal{E}$.

Substituting for $V_{i_{s_{i}} t_{i}}$ in the second of (9) and using (7) and (11), we obtain

$$
\begin{aligned}
V_{s t}=\frac{\lambda^{2} \varepsilon}{8} \delta_{s t} & {\left[\frac{\pi^{2} d}{6}-\sum_{i=1}^{d} \frac{1}{\left(s_{i}+1\right)^{2}}\right] } \\
+ & \frac{\lambda^{2} \varepsilon}{2} \delta_{\alpha_{s t}, d-1} \sum_{i=1}^{d}\left\{\left[\frac{\left(1-\delta_{s_{i} t_{i}}\right)}{\left(s_{i}-t_{i}\right)^{2}+\delta_{s_{i} t_{i}}}-\frac{\left(1-\delta_{s_{i} t_{i}}\right)}{\left(s_{i}+t_{i}+2\right)^{2}}\right] \cos ^{2}\left[\left(s_{i}-t_{i}\right) \frac{\pi}{2}\right]\right\} .
\end{aligned}
$$

We therefore see that off-diagonal survival of $V_{s t}$ is possible (but not guarranteed due to the presence of the $\cos ^{2}$ term) only if $\alpha_{s t}=d-1$, that is only if there exists a $k \in[1, d]$ such that $s_{i}=t_{i}$ if $i \neq k$ but $s_{k} \neq t_{k}$, so that the vectors $\mathbf{r}$ and $\mathbf{s}$ differ only at the $k^{\text {th }}$ entry.

Thus,

where

$$
\begin{aligned}
V_{s t}=\frac{\lambda^{2} \varepsilon}{8} \delta_{s t} & {\left[\frac{\pi^{2} d}{6}-\sum_{i=1}^{d} \frac{1}{\left(s_{i}+1\right)^{2}}\right] } \\
+ & \frac{\lambda^{2} \varepsilon}{2} \delta_{\alpha_{s t}, d-1}\left[\frac{1}{\left(s_{k}-t_{k}\right)^{2}}-\frac{1}{\left(s_{k}+t_{k}+2\right)^{2}}\right] \cos ^{2}\left[\left(s_{k}-t_{k}\right) \frac{\pi}{2}\right],
\end{aligned}
$$

$$
k=\sum_{j=1}^{d} j\left(1-\delta_{s_{j} t_{j}}\right)=\sum_{j=1}^{d} j\left(1-\alpha_{j_{s t}}\right)
$$

\section{Representation for $H$}

Adding the matrix elements $T_{s t}$ and $V_{s t}$ we find that the matrix elements for the Hamiltonian of the $d$-dimensional oscillator, with the direct product of eigenstates of the particle in a one dimensional box as basis, are given by

$$
\begin{aligned}
H_{s t}=\varepsilon \delta_{s t} & \sum_{i=1}^{d}\left(s_{i}+1\right)^{2}+\frac{\lambda^{2} \varepsilon}{8} \delta_{s t}\left[\frac{\pi^{2} d}{6}-\sum_{i=1}^{d} \frac{1}{\left(s_{i}+1\right)^{2}}\right] \\
+ & \frac{\lambda^{2} \varepsilon}{2} \delta_{\alpha_{s t}, d-1}\left[\frac{1}{\left(s_{k}-t_{k}\right)^{2}}-\frac{1}{\left(s_{k}+t_{k}+2\right)^{2}}\right] \cos ^{2}\left[\left(s_{k}-t_{k}\right) \frac{\pi}{2}\right],
\end{aligned}
$$

where 


$$
k=\sum_{j=1}^{d} j\left(1-\delta_{s_{j} t_{j}}\right)=\sum_{j=1}^{d} j\left(1-\alpha_{j_{s t}}\right)
$$

\section{APPLICATION: APPROXIMATE ANALYTIC EXPRESSION FOR THE ENERGY SPECTRUM OF THE 1-DIMENSIONAL CHO}

Based on our discussion in the previous sections culminating in the derivation of the explicit matrix elements of the $d$-dimensional confined harmonic oscillator, it is now straightforward, in principle, to find the eigenvalues of the oscillator. In practice however, the quantization remains a formidable task because of the exponential growth of the size of the Hamiltonian matrix with $d$. However, since the matrix elements are available in explicit form, they can be gainfully employed, for example in perturbation calculations, to obtain approximate analytical results.

It is our aim in the remaining part of this paper to derive an approximate analytic expression for the energy spectrum of the 1-dimensional confined harmonic oscillator. We will treat the potential energy of the confined oscillator as a perturbation of the kinetic energy term, the latter being the exactly solvable particle in a box Hamiltonian, with the non-degenerate eigenstates given in (1). Results from perturbation calculations, in the one dimensional case, can also be found in references BAIJAL and SINGH (1955), PADNOS (1965) and GUEORGUIEV et al. (2006).

\section{Energy spectrum of the 1 - dimensional cho}

For the discussion of the confined harmonic oscillator in one dimension, it is convenient to drop the subscripts on $s$ and $t$. Also we shall refer to $H_{i}, V_{i}, T_{i}$ and $x_{i}$ simply as $H, V, T$ and $x$ respectively. The eigenvalue problem is therefore

$$
H\left|E_{r}\right\rangle=E_{r}\left|E_{r}\right\rangle, \quad r=0,1,2, \ldots, N-1 .
$$

Since the eigenstates of $T(x)$ are known, being the $\varphi_{r}(x)$ of (1), it is convenient, for small values of the classical oscillator frequency, $\omega$, to treat $V(x)$ as a perturbation of the exactly solvable particle in a box Hamiltonian, $T(x)$, with $\omega^{2}$ as the perturbation parameter.

By noting that $\delta_{\alpha_{s t}, d-1}=\delta_{\alpha_{s t}, 0}=1-\delta_{s t}$ we have

$$
\begin{aligned}
V_{s t}=\frac{\lambda^{2} \varepsilon}{8} \delta_{s t} & {\left[\frac{\pi^{2}}{6}-\frac{1}{(s+1)^{2}}\right] } \\
+ & \frac{\lambda^{2} \varepsilon}{2}\left[\frac{\left(1-\delta_{s t}\right)}{(s-t)^{2}+\delta_{s t}}-\frac{\left(1-\delta_{s t}\right)}{(s+t+2)^{2}}\right] \cos ^{2}\left[(s-t) \frac{\pi}{2}\right]
\end{aligned}
$$

and

$$
\begin{aligned}
H_{s t}=\varepsilon \delta_{s t}(s+1)^{2}+\frac{\lambda^{2} \varepsilon}{8} \delta_{s t}\left[\frac{\pi^{2}}{6}-\frac{1}{(s+1)^{2}}\right] \\
+\frac{\lambda^{2} \varepsilon}{2}\left[\frac{\left(1-\delta_{s t}\right)}{(s-t)^{2}+\delta_{s t}}-\frac{\left(1-\delta_{s t}\right)}{(s+t+2)^{2}}\right] \cos ^{2}\left[(s-t) \frac{\pi}{2}\right] .
\end{aligned}
$$

Since the states $\left|E_{r}\right\rangle$ are non-degenerate, $E_{r}$ can be approximated, using standard perturbation 
theory, as

$$
E_{r} \approx E_{r}^{(0)}+E_{r}^{(1)}+E_{r}^{(2)}+E_{r}^{(3)}
$$

The zeroth order correction to the energy of the one dimensional confined harmonic oscillator, $E_{r}^{(0)}$, being the energy of the one dimensional particle in a box and the first order correction, $E_{r}^{(1)}$, being the expectation value of the perturbation $V(x)$, in each state $\varphi_{r}(x)$, are straightforward to calculate:

$$
\begin{aligned}
E_{r}^{(0)} & =\varepsilon_{r}=\varepsilon(r+1)^{2}, \quad \text { from } \\
& \text { and } \\
E_{r}^{(1)}=V_{r r} & =\frac{\lambda^{2} \varepsilon}{8}\left[\frac{\pi^{2}}{6}-\frac{1}{(r+1)^{2}}\right], \quad \text { from } \\
& =\frac{\lambda^{2} \varepsilon}{8}\left[\zeta(2)-\frac{1}{(r+1)^{2}}\right],
\end{aligned}
$$

where $\zeta(m)$ is the Riemann zeta function defined by

$$
\zeta(m)=\sum_{r=1}^{\infty} \frac{1}{r^{m}} .
$$

The second order correction to the energy of the one dimensional confined harmonic oscillator, $E_{r}^{(2)}$, is given by

$$
E_{r}^{(2)}=\sum_{s=0}^{\infty} \frac{V_{r s} V_{s r}}{\varepsilon_{r s}}=\sum_{s=0}^{r-1} \frac{V_{r s} V_{s r}}{\varepsilon_{r s}}+\sum_{s=r+1}^{\infty} \frac{V_{r s} V_{s r}}{\varepsilon_{r s}}
$$

where

$$
\mathcal{E}_{r s}=\varepsilon_{r}-\varepsilon_{s}=\mathcal{E}(r+s+2)(r-s)
$$

so that

$$
\begin{aligned}
\frac{2 \varepsilon(r+1)}{\varepsilon_{r s}} & =\frac{1}{(r+s+2)}+\frac{1}{(r-s)} \\
& \text { and } \\
\frac{2 \varepsilon(s+1)}{\varepsilon_{r s}} & =-\frac{1}{(r+s+2)}+\frac{1}{(r-s)} .
\end{aligned}
$$

Since $V$ is a real symmetric matrix, (14) is simply

$$
E_{r}^{(2)}=\sum_{s=0}^{r-1} \frac{V_{r s}^{2}}{\varepsilon_{r s}}+\sum_{s=r+1}^{\infty} \frac{V_{r s}^{2}}{\varepsilon_{r s}} .
$$

We note that the matrix elements occuring in (17) are necessarily off-diagonal $(s \neq r)$. Furthermore the only surviving elements $V_{r s}$, according to (13), are those for which $r$ and $s$ are both odd or both even. It therefore follows from (13) that

$$
\begin{aligned}
V_{r s} & =\frac{\lambda^{2} \varepsilon}{2}\left[\frac{1}{(r-s)^{2}}-\frac{1}{(r+s+2)^{2}}\right] \cos ^{2}\left[(s-t) \frac{\pi}{2}\right] \\
& =\frac{\lambda^{2} \varepsilon}{2}\left(\frac{1}{(r-s)}-\frac{1}{(r+s+2)}\right)\left(\frac{1}{(r-s)}+\frac{1}{(r+s+2)}\right) \cos ^{2}\left[(r-s) \frac{\pi}{2}\right],
\end{aligned}
$$

and using (16) we have

$$
V_{r s}=2 \lambda^{2} \varepsilon^{3} \frac{(r+1)(s+1)}{\varepsilon_{r s}^{2}} \cos ^{2}\left[(r-s) \frac{\pi}{2}\right], \quad s \neq r .
$$

From (15) and (18) and noting that 
we have

$$
\cos ^{4}\left[(r-s) \frac{\pi}{2}\right] \equiv \cos ^{2}\left[(r-s) \frac{\pi}{2}\right]
$$

and thus (17) now becomes

$$
\frac{V_{r s}^{2}}{\varepsilon_{r s}}=4 \lambda^{4} \varepsilon \frac{(r+1)^{2}(s+1)^{2}}{(r-s)^{5}(r+s+2)^{5}} \cos ^{2}\left[(r-s) \frac{\pi}{2}\right]
$$

$$
\begin{aligned}
& E_{r}^{(2)}=4 \lambda^{4} \varepsilon(r+1)^{2} \sum_{s=0}^{r-1} \frac{(s+1)^{2}}{(r-s)^{5}(r+s+2)^{5}} \cos ^{2}\left[(r-s) \frac{\pi}{2}\right] \\
&+4 \lambda^{4} \varepsilon(r+1)^{2} \sum_{s=r+1}^{\infty} \frac{(s+1)^{2}}{(r-s)^{5}(r+s+2)^{5}} \cos ^{2}\left[(r-s) \frac{\pi}{2}\right] .
\end{aligned}
$$

Classifying the energy corrections in (19) by parity of $r$ we have

$$
E_{q}^{(2)}=4 \lambda^{4} \varepsilon(q+1)^{2}\left(A_{q}+B_{q}\right)
$$

with

$$
\begin{aligned}
A_{q} & =\sum_{s=0}^{q-1} \frac{(s+1)^{2}}{(q-s)^{5}(q+s+2)^{5}} \cos ^{2}\left[(q-s) \frac{\pi}{2}\right] \\
& \text { and } \\
B_{q} & =\sum_{s=q+1}^{\infty} \frac{(s+1)^{2}}{(q-s)^{5}(q+s+2)^{5}} \cos ^{2}\left[(q-s) \frac{\pi}{2}\right],
\end{aligned}
$$

where $q=2 r$ for even levels and $q=2 r+1$ for odd levels.

Choosing

$$
f_{s}=\frac{(s+1)^{2}}{(2 r-s)^{5}(2 r+s+2)^{5}} \cos ^{2}\left[(2 r-s) \frac{\pi}{2}\right]
$$

in the following summation identity (see section 2.1.1 of GOULD 2011) for more general formulas)

$$
\sum_{s=0}^{M} f_{s}=\sum_{s=0}^{(M-(M \bmod 2)) / 2} f_{2 s}+\sum_{s=0}^{(M+(M \bmod 2)) / 2-1} f_{2 s+1},
$$

allows us to write (noting that $f_{2 s+1}=0$ with the present choice of $f_{s}$ )

$$
\begin{aligned}
A_{2 r} & =\sum_{s=0}^{2 r-1} \frac{(s+1)^{2}}{(2 r-s)^{5}(2 r+s+2)^{5}} \cos ^{2}\left[(2 r-s) \frac{\pi}{2}\right] \\
& =\sum_{s=0}^{r-1} \frac{(2 s+1)^{2}}{(2 r-2 s)^{5}(2 r+2 s+2)^{5}} \\
& =\frac{1}{2^{10}} \sum_{s=0}^{r-1} \frac{(2 s+1)^{2}}{(r-s)^{5}(r+s+1)^{5}}
\end{aligned}
$$

and

$$
B_{2 r}=\frac{1}{2^{10}} \sum_{s=r+1}^{\infty} \frac{(2 s+1)^{2}}{(r-s)^{5}(r+s+1)^{5}} .
$$

Similarly, taking identity (21) into consideration, we have

$$
\begin{aligned}
A_{2 r+1} & =\sum_{s=0}^{2 r} \frac{(s+1)^{2}}{(2 r-s+1)^{5}(2 r+s+3)^{5}} \cos ^{2}\left[(2 r-s+1) \frac{\pi}{2}\right] \\
& =\sum_{s=0}^{r-1} \frac{(2 s+2)^{2}}{(2 r-2 s)^{5}(2 r+2 s+4)^{5}} \\
& =\frac{4}{2^{10}} \sum_{s=0}^{r-1} \frac{(s+1)^{2}}{(r-s)^{5}(r+s+2)^{5}}
\end{aligned}
$$


and

$$
B_{2 r+1}=\frac{4}{2^{10}} \sum_{s=r+1}^{\infty} \frac{(s+1)^{2}}{(r-s)^{5}(r+s+2)^{5}} .
$$

We note that the above results can be combined into

and

$$
A_{q}=\sum_{s=0}^{r-1} \frac{(2 s+1+q \bmod 2)^{2}}{(2 r-2 s)^{5}(q+2 s+2+q \bmod 2)^{5}}
$$

$$
B_{q}=\sum_{s=r+1}^{\infty} \frac{(2 s+1+q \bmod 2)^{2}}{(2 r-2 s)^{5}(q+2 s+2+q \bmod 2)^{5}},
$$

where $q=2 r$ or $q=2 r+1$.

The finite sums $A_{2 r}$ and $A_{2 r+1}$ as given in (22) and (24) as well as the infinite sums $B_{2 r}$ and $B_{2 r+1}$ as given in (23) and (25) are expressible in closed form, in terms of the well-studied polygamma functions; a computer algebra system, such as Waterloo Maple, comes in handy for this purpose. Putting the results together in (20), the final result is (see the Appendix for the Maple code)

$$
E_{r}^{(2)}=\frac{\lambda^{4} \varepsilon}{128}\left(\frac{\zeta(4)}{(r+1)^{2}}-\frac{5 \zeta(2)}{(r+1)^{4}}+\frac{7}{(r+1)^{6}}\right), \quad r=0,1,2, \ldots
$$

We remark that an equivalent result to ours, for $E_{r}^{(2)}$, can also be found in reference BAIJAL and SiNGH (1955). The sum was however left unevaluated in that paper.

In standard non-degenerate perturbation theory, the third order correction to the energy of the one dimensional confined harmonic oscillator, $E_{r}^{(3)}$, is given by

$$
E_{r}^{(3)}=\sum_{s=0}^{N} \sum_{t=0}^{N} \frac{V_{r s} V_{s t} V_{t r}}{\mathcal{E}_{r s} \mathcal{E}_{r t}}-V_{r r} \sum_{s=0}^{N} \frac{V_{r s} V_{s r}}{\mathcal{E}_{r s}^{2}} .
$$

Working exactly as in computing the second order corrections, while taking note of the following summation identity

$$
\sum_{s=a}^{N} \sum_{t=a}^{N} f_{s t}=\sum_{s=a}^{N} f_{s s}+\sum_{s=a}^{N-1} \sum_{t=s+1}^{N}\left(f_{s t}+f_{t s}\right)
$$

we find that $E_{r}^{(3)}$ is expressible in closed form, in terms of the polygamma functions. In the limit of $N \rightarrow \infty$ the result is

$$
E_{r}^{(3)}=\frac{\lambda^{6} \varepsilon}{2048}\left(\frac{\zeta(6)}{(r+1)^{4}}-\frac{60 \zeta(4)}{(r+1)^{6}}+\frac{186 \zeta(2)}{(r+1)^{8}}-\frac{242}{(r+1)^{10}}\right) .
$$

Thus, the energy corrections can be written as

where

$$
E_{r}^{(m)}=\frac{\lambda^{2 m} \varepsilon}{2^{4 m-1}} \sum_{n=0}^{m} \frac{(-1)^{n} \zeta(2 m-2 n) c_{n}^{(m)}}{\left((r+1)^{2}\right)^{m+n-1}}, \quad m=0,1,2,3,
$$




$$
\begin{array}{ll}
c_{0}^{(0)} & =-1, \\
c_{0}^{(1)} & =1, c_{1}^{(1)}=-2, \\
c_{0}^{(2)} & =1, c_{1}^{(2)}=5, c_{2}^{(2)}=-14 \\
\text { and } & \\
c_{0}^{(3)} & =1, c_{1}^{(3)}=60, c_{2}^{(3)}=186, c_{3}^{(3)}=-484 .
\end{array}
$$

To the sixth order in the classical oscillator frequency, $\omega$, therefore, the one dimensional cho has the energy spectrum

$$
E_{r} \approx \sum_{m=0}^{3} E_{r}^{(m)}=\sum_{m=0}^{3}\left\{\frac{\lambda^{2 m} \varepsilon}{2^{4 m-1}} \sum_{n=0}^{m} \frac{(-1)^{n} \zeta(2 m-2 n) c_{n}^{(m)}}{\left((r+1)^{2}\right)^{m+n-1}}\right\},
$$

with $c_{n}^{(m)}$ as given in (26).

The form of (27) allows to conjecture the existence of an exact formula for the energy spectrum of the one dimensional confined harmonic oscillator, in the form

$$
E_{r}=\sum_{m=0}^{\infty} E_{r}^{(m)}=\sum_{m=0}^{\infty}\left\{\frac{\lambda^{2 m} \mathcal{E}}{2^{4 m-1}} \sum_{n=0}^{m} \frac{(-1)^{n} \zeta(2 m-2 n) c_{n}^{(m)}}{\left((r+1)^{2}\right)^{m+n-1}}\right\}, \quad c_{n}^{(m)} \in \mathbf{Z} \backslash\{0\} .
$$

\section{SUMMARY}

We have derived an explicit matrix representation for the $d$-dimensional confined harmonic oscillator, using the eigenstates of the kinetic energy operator as basis vectors.

We showed that the Hamiltonian

$$
H(\mathbf{x})=-\frac{\hbar^{2}}{2 m} \sum_{i=1}^{d} \frac{\partial^{2}}{\partial x_{i}^{2}}+\frac{1}{2} m \omega^{2} \sum_{i=1}^{d} x_{i}^{2}, \quad\left|x_{i}\right| \leq L,
$$

has the explicit $N^{d} \times N^{d}$ matrix representation

$$
\begin{aligned}
H_{s t}=\varepsilon \delta_{s t} & \sum_{i=1}^{d}\left(s_{i}+1\right)^{2}+\frac{\lambda^{2} \varepsilon}{8} \delta_{s t}\left[\frac{\pi^{2} d}{6}-\sum_{i=1}^{d} \frac{1}{\left(s_{i}+1\right)^{2}}\right] \\
+ & \frac{\lambda^{2} \varepsilon}{2} \delta_{\alpha_{s t}, d-1}\left[\frac{1}{\left(s_{k}-t_{k}\right)^{2}}-\frac{1}{\left(s_{k}+t_{k}+2\right)^{2}}\right] \cos ^{2}\left[\left(s_{k}-t_{k}\right) \frac{\pi}{2}\right],
\end{aligned}
$$

with

and

$$
\varepsilon=\frac{\pi^{2} \hbar^{2}}{8 m L^{2}}, \lambda=\omega \hbar / \varepsilon=\varepsilon_{\omega} / \varepsilon, \alpha_{s t}=\sum_{i=1}^{d} \alpha_{i_{s t}}=\sum_{i=1}^{d} \delta_{s_{i} t_{i}}
$$

$$
k=\sum_{j=1}^{d} j\left(1-\delta_{s_{j} t_{j}}\right)=\sum_{j=1}^{d} j\left(1-\alpha_{j_{s t}}\right)
$$

where $s, t=0,1,2, \ldots, N^{d}-1$ and

$$
s_{i}=\left\lfloor\frac{s}{N^{d-i}}\right\rfloor \bmod N, \quad i=1,2, \ldots, d
$$


and

$$
t_{i}=\left\lfloor\frac{t}{N^{d-i}}\right\rfloor \bmod N, \quad i=1,2, \ldots, d .
$$

In particular, for the one-dimensional confined harmonic oscillator, we have an $N \times N$ representation with the matrix elements given by

$$
\begin{aligned}
H_{s t}=\varepsilon \delta_{s t}(s+1)^{2}+\frac{\lambda^{2} \varepsilon}{8} \delta_{s t}\left[\frac{\pi^{2}}{6}-\frac{1}{(s+1)^{2}}\right] & \\
+ & \frac{\lambda^{2} \varepsilon}{2}\left[\frac{1}{(s-t)^{2}+\delta_{s t}}-\frac{1}{(s+t+2)^{2}}\right]\left(1-\delta_{s t}\right) \cos ^{2}\left[(s-t) \frac{\pi}{2}\right],
\end{aligned}
$$

for $s, t=0,1,2, \ldots, N-1$.

Finally, we derived the following approximate analytic expression for the energy spectrum of the 1-dimensional cho, to the sixth order in the oscillator frequency $\omega$,

$$
E_{r} \approx \sum_{m=0}^{3} E_{r}^{(m)}=\sum_{m=0}^{3}\left\{\frac{\lambda^{2 m} \varepsilon}{2^{4 m-1}} \sum_{n=0}^{m} \frac{(-1)^{n} \zeta(2 m-2 n) c_{n}^{(m)}}{\left((r+1)^{2}\right)^{m+n-1}}\right\}, \quad r=0,1,2, \ldots, N,
$$

with $c_{n}^{(m)}$ as given in (26).

\section{APPENDIX}

\section{Maple code to determine $E_{r}^{(2)}$}

$>$ summand: $=\mathrm{q}->(2 * \mathrm{~s}+1+\operatorname{modp}(\mathrm{q}, 2))^{\wedge} 2 /(2 * \mathrm{r}-2 * \mathrm{~s})^{\wedge} 5 /(\mathrm{q}+2 * \mathrm{~s}+2+\operatorname{modp}(\mathrm{q}, 2))^{\wedge} 5$;

$$
\text { summand }:=q \rightarrow \frac{(2 s+1+\bmod p(q, 2))^{2}}{(2 r-2 s)^{5}(q+2 s+2+\bmod p(q, 2))^{5}}
$$

$>\mathrm{A} 2 \mathrm{r}:=\operatorname{sum}(\operatorname{summand}(2 * \mathrm{r}), \mathrm{s}=0 . . \mathrm{r}-1)$ :

\# replace the last ":" with ";" to see the polygamma sums

$>\mathrm{B} 2 \mathrm{r}:=\operatorname{sum}(\operatorname{summand}(2 * \mathrm{r}), \mathrm{s}=\mathrm{r}+1$...infinity):

\# replace ":" with ";" to see the polygamma sums

$>\mathrm{E} 2 \mathrm{r}:=\operatorname{expand}\left(\operatorname{simplify}\left(4 *(2 * \mathrm{r}+1)^{\wedge} 2 *(\mathrm{~A} 2 \mathrm{r}+\mathrm{B} 2 \mathrm{r})\right)\right)$ :

\# we suppress the factor [lambda^ $4 *$ epsilon]

$>\#$ collect terms of the same order in $\mathrm{Pi}$

E2r:=collect(\%,Pi):

$>\mathrm{E} 2 \mathrm{r}:=$ factor $\left(\operatorname{coeff}\left(\mathrm{E} 2 \mathrm{r}, \mathrm{Pi}^{\wedge} 4\right)\right) * \mathrm{Pi}^{\wedge} 4+$ factor $\left(\operatorname{coeff}\left(\mathrm{E} 2 \mathrm{r}, \mathrm{Pi}^{\wedge} 2\right)\right) * \mathrm{Pi}^{\wedge} 2+o p(3, \mathrm{E} 2 \mathrm{r})$;

$$
E 2 r:=\frac{\pi^{4}}{11520(2 r+1)^{2}}-\frac{5 \pi^{2}}{768(2 r+1)^{4}}+\frac{7}{128(2 r+1)^{6}}
$$

$>\#$ we now include the lambda^ $4 *$ epsilon

E2r:=lambda^4*epsilon*E2r;

$$
E 2 r:=\lambda^{4} \varepsilon\left(\frac{\pi^{4}}{11520(2 r+1)^{2}}-\frac{5 \pi^{2}}{768(2 r+1)^{4}}+\frac{7}{128(2 r+1)^{6}}\right)
$$

Since $\zeta(2)=\pi^{2} / 6$ and $\zeta(4)=\pi^{4} / 90$ it follows that 


$$
E_{2 r}^{(2)}=\frac{\lambda^{4} \varepsilon}{128}\left(\frac{\zeta(4)}{(2 r+1)^{2}}-\frac{5 \zeta(2)}{(2 r+1)^{4}}+\frac{7}{(2 r+1)^{6}}\right)
$$

Similarly running the above code with summand $(2 r+1)$ gives

$$
E_{2 r+1}^{(2)}=\frac{\lambda^{4} \varepsilon}{128}\left(\frac{\zeta(4)}{(2 r+2)^{2}}-\frac{5 \zeta(2)}{(2 r+2)^{4}}+\frac{7}{(2 r+2)^{6}}\right) .
$$

From (A1) and (A2) we conclude that

$$
E_{r}^{(2)}=\frac{\lambda^{4} \varepsilon}{128}\left(\frac{\zeta(4)}{(r+1)^{2}}-\frac{5 \zeta(2)}{(r+1)^{4}}+\frac{7}{(r+1)^{6}}\right) .
$$

\section{References}

[1] AL-JABER, S.M., A confined $N$-Dimensional Harmonic Oscillator, International Journal of Theoretical Physics 47 (2008) 1853-1864.

[2] Baijal, J.S., Singh, K.K., The energy-levels and transition probabilities for a bounded linear Harmonic Oscillator, Progress of Theoretical Physics 14 (3) (1955) 214-224.

[3] Campoy, G., Aquino, N., Granados, V.D., Energy eigenvalues and Einstein coefficients for the one-dimensional confined harmonic oscillators, Journal of Physics A: Mathematical and General 35 (5) (2002) 4903-4914.

[4] Gould, H.W., Table for Fundamentals of Series: Part I: Basic properties of series and products, From the seven unpublished manuscripts of H. W. Gould, 2011.

[5] Gueorguiev, V.G., Rau, A.R.P., DraAyer, J.P., Confined one-dimensional Harmonic Oscillator as a two-mode system, American Journal of Physics 74 (5) (2006) 394-403.

[6] Montgomery, H.E., JR, Campoy, G., Aquino, N., The confined N-dimensional Harmonic Oscillator revisited, Physica Scripta 81 (4) (2010) Article ID 045010, DOI: 10.1088/0031-8949/81/04/045010.

[7] PADnOs, N., Approximating the harmonic oscillator by a particle in a box, Journal of Chemical Education 42 (11) (1965) 600. 